\title{
P53 Peptide Vaccine MPS-128
}

National Cancer Institute

\section{Source}

National Cancer Institute. p53 Peptide Vaccine MPS-128. NCI Thesaurus. Code C1986.

A peptide-based cancer vaccine composed of amino acids 264 to 272 of the wild-type protein encoded by the P53 gene. p53 peptide vaccine may elicit an HLA-A2.1-restricted cytotoxic T lymphocyte immune response against tumor cells that overexpress p53 protein. (NCI04) 